\title{
HEREDABILIDAD Y VALOR GENÉTICO (REML/BLUP) EN GENOTIPOS DE UN EUCALIPTO TOLERANTE A LA SEQUÍA, EN EL NORTE DE CHILE
}

\author{
HERITABILITY AND GENETIC VALUE (REML/BLUP) IN GENOTYPES OF AN EUCALYPT \\ DROUGHT TOLERANCE, IN THE NORTHEN OF CHILE
}

\section{Freddy Mora ${ }^{1}$ \\ RESUMEN}

Escasas especies del género Eucalyptus son consideradas útiles para la forestación en zonas secas. Una de ellas es Eucalyptus cladocalyx, que además está siendo considerada en programas de hibridación interespecífica para mejorar características como la tolerancia al déficit hídrico. 49 genotipos (familias) de medio hermanos, provenientes de seis poblaciones, fueron evaluadas a los 30 meses después de la plantación, en dos localidades del semiárido Chileno: Caracas y Tunga. Cada uno de los ensayos tuvo un diseño de bloques completos al azar, con parcelas de un árbol, con 30 bloques. Se estimaron componentes de varianza por el método de máxima verosimilitud restringida (REML) en un modelo lineal mixto. Se obtuvieron los valores genéticos a través de la mejor predicción lineal no sesgada (BLUP) del efecto aleatorio debido al origen (poblaciones). A pesar de que el cálculo de la heredabilidad fue conservador al considerar un coeficiente $k=2,5$, las heredabilidades de la altura fueron moderadas y altas, con valores desde 0,48 en Caracas, a 0,76 en Tunga. Los valores de la heredabilidad para el diámetro basal fueron moderados, variando desde 0,26 a 0,39 en Caracas y Tunga, respectivamente. Los orígenes de Cowell, Remarkable e Illapel, mostraron valores genéticos negativos en ambos sitios. Por otro lado, la procedencia de Wirrabara obtuvo siempre valores genéticos superiores al resto, mostrando ser una procedencia interesante para el programa de mejoramiento de la especie. Si los valores de heredabilidad permanecen en el tiempo, permitiría obtener importantes ganancias genéticas, y con ello mejorar significativamente la productividad de las futuras plantaciones de Eucalyptus cladocalyx.

Palabras claves: componentes de varianza; modelos mixtos; semiárido.

\section{ABSTRACT}

Few species of Eucalyptus are considered useful for forestation in arid zones. Eucalyptus cladocalyx is one of them, and it has been regarded in inter-specific hybrid programs for improvement characteristics like drought tolerance. 49 half-sib families from six populations were evaluated 30 months after the plantations in two localities of Chilean semiarid: Caracas and Tunga. Each trial was a randomized complete blocks design, with single tree plots and 30 blocks. Variance components were estimated by restricted maximum likelihood (REML) method in a mixed linear model. Predicted genetic values for origin random effect ware obtained by the use of best linear best linear unbiased predictor (BLUP). Although heritability was calculated using $k$ coefficient equal to 2.5 , heritability values of height were moderated and high, with values from 0.48 in Caracas to 0.76 in Tunga. Heritability values for basal diameter were moderated, varying from 0.26 to 0.39 in Caracas and Tunga, respectively. The origins of Cowell, Remarkable and Illapel showed negatives genetic values in both sites. Wirrabara provenance has always obtained superior genetic values, showing to be an interesting provenance for improvement program of this species. If heritability values remain in the time, they will allow genetic gains and the improvement of Eucalyptus cladocalyx plantations productivity.

Keywords: variance components; mixed models; arid zones.

\section{INTRODUCCIÓN}

Eucalyptus cladocalyx (F. Muell) es considerada una especie arbórea con un significativo potencial para la forestación en la zona mediterránea semiárida de Chile, debido a su capacidad de adaptación a estos ambientes, a sus características de la madera, y a su calidad floral para producción apícola. A su vez, especial interés se ha observado en la posibilidad de utilizar esta especie como combinaciones híbridas con otras especies de interés económico, para la obtención de plantas que mejoran características tales como densidad

1. Engenheiro Florestal. M.Sc. Doutorando do Programa de Pós-graduação em Genética e Melhoramento da Universidade Estadual de Maringá, Av. Colombo, 5790, Bloco 5, Sala 1, CEP 87.020-900, Maringá (PR). fmora@universiabrasil.net

Recebido para publicação em 7/04/2003 e aceito em 2/05/2006. 
de la madera y la tolerancia al déficit hídrico (Harwood et al. 2001; Mora, et al. 2005; Potts et al. 2001).

El éxito de Eucalyptus cladocalyx en programas de forestación se ha comprobado en todo el sur de Australia, Sudáfrica, África del Norte, España, Chile y Portugal, en donde existen plantaciones en áreas de suelos pobres y con escasa precipitación. Los usos de esta especie son principalmente la protección de suelos contra la erosión, producción de postes, cortinas cortavientos, ornamentación y producción de miel (Mora y Perret, 2002).

Tanto en aspectos de forestación con la especie como en su utilización en los programas de hibridación, es necesario obtener grupos de árboles que demuestren características sobresalientes, a objeto de destinar árboles más tolerantes a las condiciones semiáridas, y que expresen una rápida ocupación del espacio disponible, aspecto fundamental en ambientes desfavorables. Diversos parámetros son estimados con el fin de determinar el grado del control genético que puede presentar alguna característica de interés. Entre las estimaciones más relevantes se encuentra la heredabilidad, la cual expresa la proporción relativa de las influencias genéticas y ambientales, en la manifestación fenotípica de los caracteres, e indica, por lo tanto, el grado de facilidad o dificultad para mejorar determinados caracteres. Así, caracteres con heredabilidades bajas demandarán métodos más elaborados que aquellos con heredabilidad alta (Resende, 2002).

Tanto la predicción de valores genéticos como los métodos de selección dependen esencialmente de estimaciones verdaderas de componentes de varianza, los cuales son considerados conocidos en los procedimientos de mejor predicción (BP), mejor predicción lineal (BLP) y mejor predicción lineal no sesgada (BLUP), que en la práctica deben ser estimados con la mayor precisión posible (Resende, 2002). Patterson y Thompson (1971) desarrollaron el método de máxima verosimilitud restringida (REML) para la estimación de componentes de varianza sin sesgo, y, tal como el método de máxima verosimilitud (ML), acepta solo valores positivos de las varianzas. Farias-Neto y Resende (2001), sostienen que los procedimientos óptimos de estimación y predicción se pueden resumir en análisis de varianza e índice multiefectos para los casos balanceados, y REML y BLUP, respectivamente, los cuales se pueden aplicar en casos balanceados y no balanceados.

Searle et al. (1992) sostiene que los componentes de varianza se deben estimar con la mayor precisión posible, que tratándose de modelos lineales mixtos, con datos no balanceados, es el procedimiento de máxima verosimilitud restringida. Marcelino y Iemma (2000) y Perri y Iemma (1999) mostraron en forma práctica como pueden ser obtenidos los componentes de varianza a través de los métodos disponibles en el procedimiento MIXED de SAS ${ }^{\circledR}$, incluyendo REML, en análisis de experimentos con datos no balanceados de modelos mixtos. Así, los objetivos de este trabajo fueron estimar componentes de varianza y heredabilidad de la altura y diámetro basal, utilizado máxima verosimilitud restringida, en dos ensayos de progenie de Eucalyptus cladocalyx, establecidos en la Región de Coquimbo, Chile, y adicionalmente obtener la predicción de valores genéticos (BLUP), del efecto aleatorio debido al origen de las semillas.

\section{MATERIALES Y MÉTODOS}

En septiembre del año 2001, dos pruebas de progenie de 49 genotipos de Eucalyptus cladocalyx, fueron establecidas en la IV Región de Chile: 47 de ellos son provenientes de poblaciones naturales de Australia, adquiridas desde el banco de germoplasma del CSIRO-Australia (Australian's Commonwealth Scientific and Industrial Research Organization), y 2 son provenientes de fuentes de semilla nacional, proporcionadas por el banco de germoplasma de INFOR-Chile (Instituto de Investigación Forestal) (Tabla 1). 
TABLA 1: Localización geográfica original de los genotipos de Eucalyptus cladocalyx, incluidas en cada ensayo, en la Provincia del Choapa, Región de Coquimbo, Chile.

TABLE 1: Geographic location of the origins of Eucalyptus cladocalyx genotype included in the trials of the Province of Choapa, Coquimbo Region, Chile.

\begin{tabular}{|c|c|c|}
\hline Lugar/Origen & Latitud (Lat S) & Longitud (Long W) \\
\hline \multicolumn{3}{|l|}{ Australia: } \\
\hline Cowell & $33^{\circ} 38^{\prime} 30^{\prime \prime}$ & $136^{\circ} 40^{\prime} 58^{\prime \prime}$ \\
\hline Flinders Chase & $35^{\circ} 57^{\prime} 00^{\prime \prime}$ & $136^{\circ} 42^{`} 00^{\prime \prime}$ \\
\hline Remarkable & $32^{\circ} 43^{\prime} 00^{\prime \prime}$ & $138^{\circ} 06^{\prime} 00^{\prime \prime}$ \\
\hline Marble Range & $34^{\circ} 30^{\prime} 09^{\prime \prime}$ & $135^{\circ} 30^{\prime} 44^{\prime \prime}$ \\
\hline Wirrabara & $33^{\circ} 06^{\prime} 10^{\prime \prime}$ & $138^{\circ} 14^{\prime} 04^{\prime \prime}$ \\
\hline \multicolumn{3}{|l|}{ Chile: } \\
\hline Illapel IV Región & $31^{\circ} 40^{\prime} 46^{\prime \prime}$ & $071^{\circ} 14^{\prime} 55^{\prime \prime}$ \\
\hline
\end{tabular}

Los ensayos fueron establecidos en las Comunas de Illapel y Los Vilos, ambos Provincia de Choapa, en terrenos de la Comunidad Agrícola Tunga Norte (31 3827 Lat S; $71^{\circ} 1940$ Long W; $297 \mathrm{~m}$ de altitud) y la Hacienda Agrícola Caracas ( $31^{\circ} 5505$ Lat S; $71^{\circ} 2710$ Long W; $167 \mathrm{~m}$ de altitud) (Tabla 2). Cada uno de los ensayos tiene un diseño de bloques completos al azar, con parcelas de un árbol (Single Tree Plots), con 30 bloques. Todos los árboles fueron plantados a un espaciamiento de $2 \mathrm{~m}$ x $3 \mathrm{~m}$, en cada sitio.

TABLA 2: Características climáticas de los sitios de ensayo de las progenies de Eucalyptus cladocalyx en Coquimbo, Chile.

TABLE 2: Climatic characteristic of Eucalyptus cladocalyx test sites in Coquimbo, Chile.

\begin{tabular}{|c|c|c|c|c|c|c|c|c|c|}
\hline \multirow[t]{2}{*}{ Ensayo } & \multirow[t]{2}{*}{ Comuna } & \multirow{2}{*}{$\begin{array}{c}\mathrm{DA}^{*} \\
\text { (N. y ombre) }\end{array}$} & \multirow{2}{*}{$\begin{array}{c}\text { Ubicación } \\
\text { General }\end{array}$} & \multirow{2}{*}{$\begin{array}{l}\text { PMA } \\
(\mathrm{mm})\end{array}$} & \multirow{2}{*}{$\begin{array}{c}\text { EA } \\
(\mathrm{mm})\end{array}$} & \multirow{2}{*}{$\begin{array}{l}\text { TMA } \\
\left({ }^{\circ} \mathrm{C}\right)\end{array}$} & \multirow{2}{*}{$\begin{array}{c}\text { PLH } \\
\text { (días) }\end{array}$} & \multicolumn{2}{|c|}{ Temperatura $\left({ }^{\circ} \mathrm{C}\right)$} \\
\hline & & & & & & & & 1 & 2 \\
\hline $\begin{array}{l}\text { Hacienda } \\
\text { Caracas }\end{array}$ & $\begin{array}{l}\text { Los } \\
\text { Vilos }\end{array}$ & $\begin{array}{l}\text { III/Amolanas } \\
\text { - Los Vilos }\end{array}$ & Litoral & 201,1 & $1100-1200$ & 14,1 & 345 & 7,0 & 24,1 \\
\hline $\begin{array}{l}\text { Tunga } \\
\text { Norte } \\
\end{array}$ & Illapel & XIII/Illapel & Interior & 243,7 & $1200-1300$ & 15,0 & 320 & 4,6 & 27,7 \\
\hline
\end{tabular}

Donde: DA = Distrito Agroclimático; PMA = Precipitación Media Anual; EA = Evapotranspiración Anual, TMA = Temperatura Media Anual; PLH = Período Libre de Heladas; * = Caldentey (1987); 1 = mínima media del mes más frío, julio; 2 = máxima media del mes más cálido, enero.

Todas las progenies fueron originadas de polinización abierta. Los caracteres de crecimiento analizados fueron altura total y diámetro basal (diámetro a la altura del cuello de la planta), medidos a los 30 meses de edad. Adicionalmente, la tasa de crecimiento absoluta (AG) fue analizada para cada característica de crecimiento, para el período 12-30 meses, basado en la siguiente ecuación:

$A G=\frac{g_{2}-g_{1}}{t_{2}-t_{1}}$

Donde: $\mathrm{g}_{2}$ y $\mathrm{g}_{1}$ representa al crecimiento (altura o diámetro) medidos en los tiempos $\mathrm{t}_{2}(2,5$ años $)$ y $\mathrm{t}_{1}$ (1 año) respectivamente. mixto:

Para cada ensayo, análisis de sitios individuales fueron realizados usando el siguiente modelo lineal

$Y_{j k}=\mu+B_{i}+P_{j}+F_{k}+\varepsilon_{i j k}$

Donde: $\mathrm{Y}_{\mathrm{ijk}}$ representa el valor observado del k-ésimo árbol de la k-ésima familia de la j-ésima procedencia, en el i-ésimo bloque o replica, $\mu$ es la constante general, $\mathrm{B}_{\mathrm{i}}$ representa el efecto fijo de la $\mathrm{i}$ ésima replica, $P_{j}$ representa el efecto aleatorio de la j-ésima procedencia $\left(\operatorname{NID}\left(0, \sigma_{p}^{2}\right)\right), F_{k}$ representa el efecto aleatorio de la k-ésima familia $\left(\operatorname{NID}\left(0, \sigma_{\mathrm{f}}^{2}\right)\right)$, e $\mathrm{i}_{\mathrm{ijk}}$ representa el efecto residual aleatorio $\left(\left(\operatorname{NID}\left(0, \sigma_{\mathrm{e}}^{2}\right)\right)\right.$, y consiste en la interacción procedencia-bloque, aleatoria, confundidos con el error experimental. Matricialmente, el vector de observaciones $y$ tendrá una distribución normal multivariada, con promedio $\mathrm{X} \beta$ y la siguiente matriz de varianzas y covarianzas (V) (Perri y Iemma, 1999):

$V=Z_{1} G Z_{1}^{T}+Z_{2} P Z_{2}^{T}+R$ 
Donde: $\mathrm{G}, \mathrm{P}$ y $\mathrm{R}$ representan las matrices de varianzas y covarianzas de $a, p$, y $e$, respectivamente. La mejor predicción lineal no sesgada (BLUP) de los efectos aleatorios de el modelo mixto propuesto se obtiene a través de la maximización de la función de densidad de probabilidad conjunta, considerando los efectos fijos y aleatorios, la cual fue determinada para el efecto aleatorio de las procedencias, vía ecuaciones de los modelos lineales mixtos (Henderson, 1984; Martins et al. 1997).

Para las estimaciones de componentes de varianza y las soluciones de los efectos aleatorios de las procedencias, se utilizó el procedimiento MIXED de SAS ® 8.02 (SAS Institute, 1996), opción REML (Máxima Verosimilitud Restringida). Tal estimación considera un algoritmo de Newton-Raphson.

Estimaciones de heredabilidad (sensu stricto) fueron obtenidas para los caracteres de crecimiento, utilizando la siguiente expresión:

$h_{a}^{2}=\frac{k \sigma_{f}^{2}}{\sigma_{\rho}^{2}}$

Donde $\sigma_{\mathrm{f}}^{2}$ representa la varianza familiar, y $\sigma_{\mathrm{e}}^{2}$ representa la varianza residual. La fracción del numerador corresponde a la varianza aditiva $\left(\sigma_{\mathrm{a}}^{2}\right)$. La fracción del denominador corresponde a la varianza fenotípica $\left(\sigma_{p}^{2}\right)$.

La existencia de autopolinización puede insesgar la estimación de los componentes de varianza aditiva en poblaciones de árboles forestales polinizados en forma natural (Squillace, 1974). McDonald et al. (2003), estudió la autopolinización y divergencia genética de Eucalyptus cladocalyx, encontrando que las tasas de exocruzamiento, en poblaciones naturales, pueden variar desde 0 a $100 \%$, y que una proporción relativamente grande de árboles por población (entre 14 y 50\%), produjo semillas originadas por autopolinización. Considerando estos resultados, en el presente estudio se utilizó un coeficiente $k$ de 2,5 (Potts et al. 1995). Por lo tanto, la varianza aditiva fue estimada a partir de la siguiente ecuación:

$\sigma^{2}=2,5 * \sigma^{2}$

El error estándar (e.e.) de la heredabilidad fue estimado de acuerdo a la expresión estimada por Falconer (1981):

$$
e e\left(h_{a}^{2}\right)=\frac{32 h_{a}^{2}}{n b f}
$$

Donde $n, b$ y $f$ representan el número de repeticiones por parcela, número de bloques, y número de progenies testadas, respectivamente.

\section{RESULTADOS Y DISCUSIÓN}

El análisis de varianza mostró diferencias significativas $(\mathrm{p}<0,01)$ en la varianza familiar y debido a las procedencias, para todos los caracteres de crecimiento en cada sitio. Parra y Chung (1997) analizaron un ensayo de procedencias de Eucalyptus cladocalyx de 31 meses de edad, en un sitio cercano al ensayo Caracas del presente estudio, y encontraron diferencias significativas entre los genotipos, para los caracteres de altura y diámetro basal.

Las estimaciones de la heredabilidad para el diámetro basal fueron moderadas, variando desde 0,26 en el sitio de Caracas, hasta 0,39 en Tunga.

A pesar de que el cálculo de la heredabilidad fue conservador al considerar un coeficiente $k=2,5$ (Potts et al. 1995), las heredabilidades de la altura fueron moderadas y altas, con valores desde 0,48 en Caracas, a 0,76 en el sitio de Tunga. Los valores de heredabilidades de cada característica fueron siempre mayores en el sitio de Tunga, lo que podría estar evidenciando un mayor control genético aditivo del crecimiento de las progenies en el sitio interior. Namkoong et al. (1972) sostiene que la magnitud de las varianzas genéticas y fenotípicas de los caracteres de crecimiento cambian con el tiempo. Por lo tanto, es obvio que los cuocientes entre estos dos componentes (heredabilidad) también podrían cambiar.

Los valores altos de heredabilidad en altura indican que es posible realizar métodos simples de mejoramiento (Resende, 2002). A su vez, la alta heredabilidad encontrada es propia de material proveniente de poblaciones naturales, donde es posible encontrar y capturar mayor variabilidad genética interpoblacional. 
TABLA 3: Componentes de varianza y heredabilidad $\left(\mathrm{h}^{2}\right)$ estimados de los ensayos de progenie de Eucalyptus cladocalyx en Coquimbo, Chile.

TABLE 3: Variance components and heritability $\left(\mathrm{h}^{2}\right)$ estimated from the progeny trials of Eucalyptus cladocalyx in Coquimbo, Chile.

\begin{tabular}{l|r|r|r|r|r|c}
\hline Sitio / Carácter & \multicolumn{1}{c}{$\sigma_{\mathrm{f}}^{2}$} & \multicolumn{1}{c}{$\sigma_{\mathrm{p}}^{2}$} & \multicolumn{1}{c}{$\sigma_{\mathrm{e}}^{2}$} & Varianza Aditiva & Varianza Fenotípica & $\mathrm{h}^{2} \pm$ e.e. \\
\hline Caracas: & & & & & & \\
H30 & 389,19 & 216,89 & 1414,37 & 972,98 & 2020,45 & $0,48 \pm 0,010$ \\
D30 & 13,61 & 5,06 & 103,97 & 34,02 & 122,64 & $0,28 \pm 0,006$ \\
H12-30 & 176,93 & 65,40 & 621,23 & 442,32 & 863,56 & $0,51 \pm 0,011$ \\
D12-30 & 5,53 & 1,99 & 45,19 & 13,81 & 52,70 & $0,26 \pm 0,006$ \\
Tunga: & & & & & & \\
H30 & 1341,49 & 550,66 & 2838,26 & 3353,73 & 4730,41 & $0,71 \pm 0,015$ \\
D30 & 31,07 & 5,43 & 162,40 & 77,68 & 198,90 & $0,39 \pm 0,009$ \\
H12-30 & 598,39 & 196,29 & 1178,16 & 1495,98 & 1972,84 & $0,76 \pm 0,017$ \\
D12-30 & 11,02 & 1,95 & 66,01 & 27,55 & 78,98 & $0,35 \pm 0,008$ \\
\hline
\end{tabular}

Donde: e.e. $=$ error estándar; $\sigma_{\mathrm{f}}^{2}, \sigma_{\mathrm{p}}^{2}, \mathrm{y} \sigma_{\mathrm{e}}^{2}=$ varianza familiar, de procedencias y residual, respectivamente; H30 y D30 = altura y diámetro medidos a los 30 meses; H12-30 y H12-30 = tasa de crecimiento en altura y diámetro del periodo de 12 a 30 meses.

El error estándar de la heredabilidad fue similar en ambos sitios debido a que igual número de progenies por familia fueron medidas en los ensayos. La varianza residual $\left(\sigma^{2}\right)$ fue la mayor fuente de variación en ambos ensayos. La magnitud relativa de esta componente (expresada en porcentaje de la variación total) varió desde 59,7\% para la tasa de crecimiento absoluto de la altura del sitio Tunga, a 85,7\% para la tasa de crecimiento absoluto del diámetro del sitio Caracas. La magnitud relativa de la varianza residual fue siempre menor en Tunga, mostrando una relación inversa con los valores de heredabilidad. Borralho et al. (1992) encontró que la varianza residual fue la mayor fuente de variación, en dos ensayos de progenie de polinización abierta de Eucalyptus globulus, promediando entre 88 y $91 \%$ de la variación total del carácter altura.

En la Tabla 4 se muestran los valores genéticos predichos por procedencia, localidad y característica. Las procedencias de Cowell, Remarkable e Illapel IV-R, mostraron siempre valores genéticos negativos en ambos sitios, el valor más bajo fue encontrado para el valor genético de Cowell $(-23,3)$ correspondiente a la característica de altura medido a los 30 meses en Tunga. Por otro lado, la procedencia de Wirrabara mostró siempre valores genéticos superiores al resto, en ambas características y sitios, entregando un valor máximo en el sitio de Tunga (25,5). Parra y Chung (1997), encontraron que los genotipos provenientes de las localidades de Wirrabara, mostraron una mejor respuesta del crecimiento, en comparación con otras procedencias, siendo similar su desarrollo a las procedencias locales, correspondientes a las localidades de Longotoma ( $\left.32^{\circ} 24^{\prime} \mathrm{S} ; 71^{\circ} 23^{\prime} \mathrm{O}\right)$, Peralillo $\left(31^{\circ} 43^{\prime} \mathrm{S} ; 71^{\circ} 11^{\prime} \mathrm{O}\right)$ y Peñuela $\left(33^{\circ} 11^{\prime} \mathrm{S} ; 71^{\circ} 33^{\prime} \mathrm{O}\right)$.

McDonald et al. (2003), sostiene que Eucalyptus cladocalyx crece naturalmente en tres zonas separadas del sur de Australia: área de Flinders Ranges, que incluye las regiones Wirrabara y Remarkable, área de Eyre Peninsula, que incluye las regiones de Cowell y Marble Range y área de Kangaroo Island, incluyendo la región de Flinders Chase. En la región de Flinders Ranges la especie alcanza un crecimiento de 35 metros, mientras que en Eyre Peninsula pocos árboles de este Eucalyptus pueden exceder los 15 metros de altura. Los árboles que crecen en Kangaroo Island presentan un crecimiento intermedio. La zona de Cowell presenta una precipitación promedio histórica de 405,0 mm/año, mientras que Wirrabara tiene un promedio de 256,6 mm/año, el cual es más cercano al valor existente en las localidades de Tunga y Caracas (Tabla 2). 
TABLA 4: Valores genéticos predichos (BLUP) de las procedencias de Eucalyptus cladocalyx evaluadas en Coquimbo, Chile.

TABLE 4: Predicted genetic values (BLUP) of Eucalyptus cladocalyx provenance evaluated in Coquimbo, Chile.

\begin{tabular}{l|rrrrrr|rrrrr}
\hline Procedencia & \multicolumn{4}{|c|}{ Caracas } & \multicolumn{4}{c}{ Tunga } \\
\cline { 2 - 10 } & \multicolumn{1}{|c|}{ H30 } & \multicolumn{1}{|c}{ D30 } & H12-30 & D12-30 & H30 & D30 & H12-30 & D12-30 \\
\hline Remarkable & $-15,1$ & $-2,3$ & $-7,2$ & $-1,5$ & $-18,5$ & $-1,6$ & $-10,3$ & $-1,0$ \\
Cowell & $-12,3$ & $-1,2$ & $-7,5$ & $-0,8$ & $-23,3$ & $-1,4$ & $-13,8$ & $-0,7$ \\
Marble Range & 7,0 & 1,8 & 2,8 & 1,0 & 7,8 & 0,9 & 5,3 & 0,6 \\
Wirrabara & 18,4 & 2,3 & 8,3 & 1,5 & 25,5 & 2,0 & 14,4 & 1,2 \\
Flinders Chase & 5,0 & $-0,1$ & 5,3 & 0,1 & 16,3 & 1,5 & 9,2 & 0,8 \\
Illapel IV Región & $-3,1$ & $-0,6$ & $-1,7$ & $-0,4$ & $-7,7$ & $-1,4$ & $-4,8$ & $-0,9$ \\
\hline
\end{tabular}

\section{CONCLUSIONES}

Para el crecimiento en altura, los resultados de las estimaciones de heredabilidad mostraron un alto grado de control genético aditivo en las progenies. A su vez, las heredabilidades del carácter diámetro fueron de moderado control genético. Los valores altos de heredabilidad en altura indican que es posible iniciar un programa de mejoramiento con métodos simples, y revela la gran variabilidad genética interpoblacional encontrada en el lugar de origen. Las heredabilidades dependen del sitio, indicando un mayor control genético aditivo del crecimiento en altura y diámetro en la ubicación interior.

Las procedencias Australianas de Cowell y Remarkable, y la procedencia Chilena Illapel IV-R, mostraron valores genéticos predichos negativos, en ambos sitios y en todas las características de crecimiento. Por el contrario, la procedencia Australiana de Wirrabara obtuvo siempre valores genéticos predichos superiores al resto, mostrando ser una procedencia interesante para el programa de mejoramiento de la especie.

Si los valores de heredabilidad permanecen en el tiempo, permitirían obtener importantes ganancias genéticas, y con ello mejorar significativamente la productividad de las futuras plantaciones de Eucalyptus cladocalyx.

\section{AGRADECIMIENTOS}

Al Fondo de Desarrollo e Innovación (FDI) al Instituto de Investigación Forestal (INFOR), a la Hacienda Agrícola Caracas y la Comunidad Agrícola de Tunga Norte, quienes co-financiaron la presente investigación perteneciente al proyecto: Desarrollo forestal maderero para la región árida de Chile, y que formó parte de la tesis de Magíster del autor, del Departamento de Biología de la Universidad de La Serena, Chile. Al profesor Dr. Raúl Moreno por las orientaciones técnicas realizadas al estudio.

\section{REFERENCIAS BIBLIOGRAFICAS}

BORRALHO, N.; ALMEIDA, M.; COTTERILL; P. Genetic control of growth of Eucalyptus gobulus in Portugal. Silvae Genetica v. 41, n. 2, p. 100-105, 1992.

CALDENTEY, P. Distritos Agroclimáticos de la IV Región. Santiago: CONAF/PNUD/FAO, 1987. 68p.

FALCONER, D.S. Introduction to quantitative genetics. 2. ed. London : Longman, 1981. 340p.

FARIAS-NETO, J.T.; RESENDE, M.D.V. Aplicação da metodologia de modelos mistos (REML/BLUP) na estimação de componentes de variância e predição de valores genéticos em pupunheira (Bactris gasipaes L). Revista Brasileira de Fruticultura, v. 23, n. 2, p. 320-324, 2001.

HARWOOD, C.; BULMAN, P.; BUSH, D.; MAZANEC, R.; STACKPOLE, D. Australian low rainfall tree improvement group. Compendium of hardwood breeding strategies. Kingston : RIRDC Press, 2001. 148p.

HENDERSON, C.R. Applications of linear models in animal breeding. Ontario : University of Guelph, 1984. 462p.

MARCELINO, S.D.R.; IEMMA, A.F. Métodos de estimação de componentes de variância em modelos mistos desbalanceados. Scientia Agricola, v. 57, n. 4, p. 643-652, 2000.

MARTINS, E.N.; LOPES, P.S.; SILVA, M.A.; JUNIOR, R.A.A.T. Uso de modelos mistos na avaliação genética animal. Viçosa : UFV, 1997. 121p. 
MCDONALD, M.W.; RAWLlingS, M.; BUTCHER, P.A.; BELL, J.C. Regional divergence and inbreeding in Eucalyptus cladocalyx (Myrtaceae). Australian Journal of Botany, v. 51, n. 4, p. 393-403,2003.

MORA, F.; PERRET, S. Eucalyptus cladocalyx en la Región de Coquimbo: especie maderera para el negocio frutícola. Revista Tierra Adentro, v. 46, p. 28-29, 2002.

MORA, F.; PALMA-ROJAS, C.; JARA-SEGUEL, P. Comparación del Cariotipo de Eucalyptus globulus y Eucalyptus cladocalyx (Myrtaceae). Agricultura Técnica (Chile), v. 65, n. 1, p. 20-25, 2005.

NAMKOONG, G.; USANIS, R.A.; SILEN, R.R. Age related variation in genetic control of height growth in douglasfir. Theoretical and Applied Genetics, v. 42, p. 151-159, 1972.

PARRA, P.; CHUNG, P. Comportamiento de procedencias de Eucalyptus cladocalyx F. Muell. de 31 meses de edad en Los Vilos, IV Región. In: FORESTACIÓN Y SILVICULTURA EN ZONAS ARIDAS Y SEMIÁRIDAS DE CHILE, Santiago, 1997. Actas... Santiago: INFOR, 1997. p. 153-160.

PATTERSON, H.D.; THOMPSON, R. Recovery of inter-block information when blocks sizes are unequal. Biometrika, v.58, p.545-554, 1971.

PERRI, S.H.V.; IEMMA, A.F. Procedimento MIXED do SAS para análise de modelos mistos. Scientia Agricola, v.56, p.959-967, 1999.

POTTS, B.M.; BARBOUR, R.C.; HINGSTON, A.B. Genetic pollution from farm forestry: using Eucalypt species and hybrids. Kingston: RIRDC Press, 2001, 108p.

RESENDE, M.D.V. Genética biométrica e estatística no melhoramento de plantas perenes. Brasília: EMBRAPA Informação Tecnológica, 2002. 975p.

SAS Institute. Statistical analysis system: user's guide. Cary : SAS Institute, 1996. 956p.

SEARLE, S.R.; CASELLA, G.; MCCULLOCH, C.E. Variance components. New York: J. Wiley, 1992. 528p.

SQUILLACE, A.E. Average genetic correlations among offspring from open-pollinated forest trees. Silvae Genetica v. 23, p. 149-156, 1974. 